\title{
International observational campaign of the 2014 eclipse of EE Cephei ${ }^{\star}$
}

\author{
D. Pieńkowski ${ }^{1}$, C. Gałan ${ }^{1}$, T. Tomov ${ }^{2}$, K. Gazeas ${ }^{3}$, P. Wychudzki $^{2}$, M. Mikołajewski ${ }^{2}$, D. Kubicki ${ }^{2}$, B. Staels ${ }^{4}$,
} S. Zoła ${ }^{5,6}$, P. Pakońska ${ }^{5}$, B. Dȩbski ${ }^{5}$, T. Kundera ${ }^{5}$, W. Ogłoza ${ }^{6}$, M. Dróżdż $\dot{z}^{6}$, A. Baran ${ }^{6}$, M. Winiarski ${ }^{6}$, M. Siwak $^{6}$, D. Dimitrov ${ }^{7}$, D. Kjurkchieva ${ }^{8}$, D. Marchev ${ }^{8}$, A. Armiński ${ }^{9}$, I. Miller ${ }^{10}$, Z. Kołaczkowski ${ }^{11}$, D. Moździerski ${ }^{11}$, E. Zahajkiewicz ${ }^{11}$, P. Bruś ${ }^{11}$, A. Pigulski ${ }^{11}$, T. Smela ${ }^{9}$, E. Conseil ${ }^{12}$, D. Boyd ${ }^{13}$, G. J. Conidis ${ }^{14}$, I. Plauchu-Frayn ${ }^{15}$, T. A. Heras ${ }^{16}$, E. Kardasis ${ }^{17}$, M. Biskupski ${ }^{9}$, R. Kneip ${ }^{30}$, L. Hambálek ${ }^{18}$, T. Pribulla ${ }^{18,19,20}$, E. Kundra ${ }^{18}$, Z. Garai ${ }^{18,19,20}$, D. Rodriguez ${ }^{21}$, T. Kamiński ${ }^{22}$, F. Dubois ${ }^{23}$, L. Logie ${ }^{23}$, A. Capetillo Blanco ${ }^{32}$, P. Kankiewicz ${ }^{24}$, E. Świerczyński ${ }^{2}$, M. Martignoni ${ }^{25}$, I. Sergey ${ }^{26}$, J. Kare Trandem Qvam ${ }^{27}$, E. Semkov ${ }^{7}$, S. Ibryamov ${ }^{7,8}$, S. Peneva $^{7}$, J.-L. Gonzalez Carballo ${ }^{28}$, J. Ribeiro ${ }^{29}$, S. Dean ${ }^{30}$, G. Apostolovska ${ }^{31}$, Z. Donchev ${ }^{7}$, L. Corp ${ }^{32,33}$, P. McDonald ${ }^{32}$, M. Rodriguez ${ }^{34}$, A. Sanchez ${ }^{35}$, K. Wiersema ${ }^{36}$, E. Conseil ${ }^{32}$, J. Menke ${ }^{37}$, I. Sergey ${ }^{38}$, and N. Richardson ${ }^{39}$

(Affiliations can be found after the references)

Received 22 November 2019 / Accepted 17 January 2020

\section{ABSTRACT}

Context. EE Cep is one of few eclipsing binary systems with a dark, dusty disc around an invisible object similar to $\varepsilon$ Aur. The system is characterised by grey and asymmetric eclipses every $5.6 \mathrm{yr}$ that have significant variations in their photometric depth, ranging from $\sim 0^{\mathrm{m}} .5$ to $\sim 2$. 0 . Aims. The main aim of the observational campaign of the EE Cep eclipse in 2014 was to test the model of disc precession. We expected that this eclipse would be one of the deepest with a depth of $\sim 2^{\mathrm{m}} 0$.

Methods. We collected multicoloured observations from almost 30 instruments located in Europe and North America. These photometric data cover 243 nights during and around the eclipse. We also analyzed low- and high-resolution spectra from several instruments.

Results. The eclipse was shallow with a depth of 0.71 in the $V$ band. The multicoloured photometry illustrates small colour changes during the eclipse with a total amplitude of order $\sim+0^{\mathrm{m}} 15$ in the $B-I$ colour index. We updated the linear ephemeris for this system by including new times of minima, measured from the three most recent eclipses at epochs $E=9,10$, and 11. We acquired new spectroscopic observations, covering orbital phases around the eclipse, which were not observed in the past and increased the data sample, filling some gaps and giving better insight into the evolution of the $\mathrm{H}_{\alpha}$ and $\mathrm{Na}$ I spectral line profiles during the primary eclipse.

Conclusions. The eclipse of EE Cep in 2014 was shallower than expected, measuring 0.71 instead of $\sim 2$. 0 . This means that our model of disc precession needs revision.

Key words. binaries: eclipsing - circumstellar matter - stars: emission-line, Be

\section{Introduction}

The 11th magnitude system EE Cep (BD+552693) is a member of a rare class of binary systems, in which the eclipses are caused by a dark, dusty disc surrounding the orbiting companion. The precursor of this group is the extremely long-period $(27.1 \mathrm{yr})$ eclipsing binary system $\varepsilon$ Aur (see Guinan \& Dewarf 2002). For a long time, these two systems were the only ones in this class of systems. Nowadays, more than a dozen such systems with similar properties are known, since many researchers have reported these systems (see e.g. Lipunov et al. 2016; Garrido et al. 2016; Rattenbury et al. 2015; Kenworthy \& Mamajek 2015; Scott et al. 2014 and references in Gałan et al. 2014). Among these systems, there is a large diversity in orbital period duration - from days to decades. Most of these systems have weak observational records; frequently only one or a few eclipses have been observed so far. The interpretation of their nature is therefore often uncertain and requires verification through further observations and analysis. An example is the case of M2-29, which we ascribed into this group (Gałan et al. 2014), based on the Hajduk et al. (2008)

\footnotetext{
$\star$ Tables A1-A29 are only available at the CDS via anonymous ftp to cdsarc.u-strasbg. fr (130.79.128.5) or via http://cdsarc. u-strasbg.fr/viz-bin/cat/J/A+A/639/A23
}

analysis of a single eclipse observed by OGLE and MACHO surveys. However Miszalski et al. (2011) prove that the scenario of eclipses by dusty disc is unlikely and propose another explanation of the observed eclipse as due to condensed dust and its evaporation around the $\mathrm{R} \mathrm{CrB}$ star.

The EECep system stands out in this group with a relatively long orbital period (5.6 yr) after $\varepsilon$ Aur and TYC 2505672-1 (69.1 yr; Lipunov et al. 2016). This system has a well-documented long history of eclipses showing very unusual behaviour for over 13 orbital epochs. The research on this object dates back to the middle of the last century. It was discovered as a variable in 1952 (epoch $E=0$ ) by Romano (1956) and confirmed soon after by Weber (1956), who reported a singular observation of a change in the brightness of this system during the previous eclipse in $1947(E=-1)$. The eclipsing nature of the light curve was established with photometric observations of the subsequent events in 1958, 1964, and 1969 (Meinunger 1973). Henceforth all consecutive, primary eclipses were observed, but no traces of the secondary eclipse have ever been recorded.

The first analytical model of the system was proposed by Meinunger (1975), who assumed that the B-type primary star is eclipsed by an M-type red giant. Pulsations of the giant should cause changes in the depth of the eclipses, whereas its 

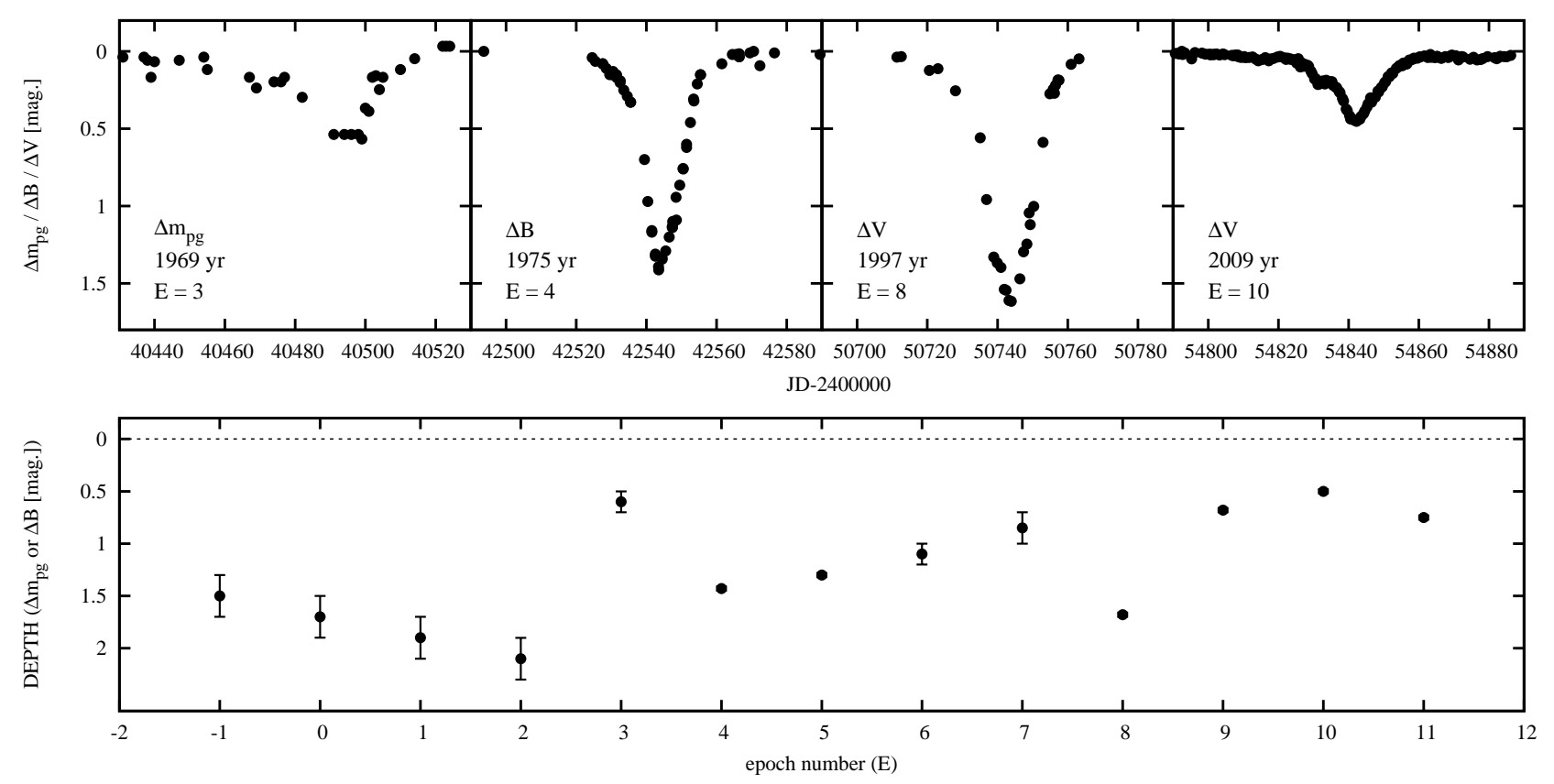

Fig. 1. Top: four representative light curves of the eclipses in EE Cep, selected among the best quality light curves with good time coverage so far: two deep ( $E=4$ and 8$)$ and two of the shallowest ( $E=3$ and 10). Bottom: time dependence relation for the depth of eclipses.

inflated atmosphere could account for atmospheric wings. This model was questioned when the first multicoloured observations using the $R$ and $I$ passbands of Johnson's photometric system were obtained in Piwnice Observatory in 1997 (Mikołajewski \& Graczyk 1999). Very small colour changes, observed during the eclipse, against the interpretation that the eclipsing component is a red giant. Mikołajewski \& Graczyk (1999) proposed another model in which the eclipses could be caused by an invisible, cold object - a dark disc around a low-luminosity central star or a close binary. From an analysis of the colour indices, these authors constrained the basic parameters of the primary component: a hot star of spectral type B5, effective temperature $14300 \mathrm{~K}$, radius $10 R_{\odot}$, and distance of $2.75 \mathrm{kpc}$. Gaia DR2 (Gaia Collaboration 2016, 2018) brought an independent parallax $\pi=(0.503 \pm 0.032) \times 10^{-3}$ arcsec, indicating that EE Cep system is closer, at $1.99 \pm 0.13 \mathrm{kpc}$.

The most striking feature of the EE Cep minima is the significant variation in their depth, from $\sim 0.5$ to $\sim 2 \mathrm{~m} 0$ (Fig. 1 ). The top panel of Fig. 1 shows the representative light curves of four minima: typical examples of deep minima observed in $1975(E=4)$ and $1997(E=8)$, the shallowest eclipse observed in detail (sor far) during 2008/9 $(E=10)$, and the exceptional eclipse with a flat bottom during its central part in $1969(E=3)$. The bottom panel of the same figure shows the variation in time of the eclipses The changes in the depth are accompanied by variations of the total duration of the eclipses (Graczyk et al. 2003). All eclipses are characterised by a similar asymmetry; the descending branches are longer than ascending branches. It is possible to distinguish in light curves repeatable phases during the eclipses: more or less sloped-bottomed transit during the central part of the eclipse is preceded and followed by the real ingress and egress, and atmospheric wings caused by semitransparent external parts of the eclipsing body. To explain this unusual behaviour, Mikołajewski \& Graczyk (1999) suggested that the eclipses are caused by the disc, which is opaque in its interior and semi-transparent in outer regions and has a varying inclination to the line of sight during different eclipses owing to precession. In this scenario, the unique flat-bottomed eclipse, observed in $1969(E=3)$, can be explained by a nearly edge-on and non-tilted projection of the disc.

To test this model we needed good temporal coverage and high quality photometric and spectroscopic observations. Obtaining dense enough sampling of the eclipses would be possible only through the collective effort of a large number of observers in various locations around the world. For the events in 2003 and 2008-2009, we organised extensive, international observing campaigns (Mikołajewski et al. 2003; Gałan et al. 2008), which attracted the attention of several dozens of observers. Analysis of the high-resolution spectra of these campaigns (Gałan et al. 2012) confirmed that the primary component is a B5III star rotating at very high velocity $\left(\sim 350 \mathrm{~km} \mathrm{~s}^{-1}\right)$, which causes inhomogeneous temperature and flux distribution on its surface as a consequence of the von Zeipel (1924) effect. Recent photometric results complemented by the historical light curves (Graczyk et al. 2003) were used to calculate a model of the system that would be able to reproduce the changing shapes of the eclipses caused by the disc precession, taking into account a mechanism which would explain the unusually different eclipses at epochs $E=3$ and 8 (see Fig. 1 - bottom). The best solution obtained for the disc precession was $P_{\text {prec }} \sim 11-12 P_{\text {orb }}$ (Gałan et al. 2012). According to this model, the eclipse in $2014(E=11)$ should attain a large, close to 2 . 0 . This model was based on observations obtained in a time interval almost exactly equal to the derived precession period. Additional photometric and spectroscopic observations were needed for its verification.

In this paper, we present photometric and spectroscopic data obtained with a new campaign, which constitutes a basis for future papers with quantitative analysis. Section 2 describes technical details of the campaign in 2014, including a compilation of instruments involved in the observations and a description of techniques used to combine data from various photometric systems. Section 3 presents the results of the campaign, i.e. the light curves and spectra. A brief discussion of the conclusions from obtained results is made in Sect. 4. 
Table 1. All instruments and their involvement in photometric observations of the EE Cep eclipses in 2014.

\begin{tabular}{|c|c|c|c|c|c|c|c|}
\hline Observatory & Country & Telescope type & Diameter [m] & Bands & $N_{\mathrm{i}}$ & $N_{\mathrm{n}}$ & Table \\
\hline Astrolab Iris & Belgium & Newton & 0.68 & $V$ & 25 & 25 & A16 \\
\hline Athens & Greece & Cassegrain & 0.4 & $B V(R I)_{C}$ & 10344 & 65 & TA1 \\
\hline Białków & Poland & Cassegrain & 0.6 & $B V(R I)_{C}$ & 150 & 38 & A5 \\
\hline Cerro del Viento & Spain & Schmidt-Cassegrain & 0.2 & $V$ & 9 & 9 & A23 \\
\hline France & France & Home made & 0.203 & $B V R$ & 5 & 2 & A24 \\
\hline Furze Hill & United Kingdom & Schmidt-Cassegrain & 0.35 & $B V(R I)_{C}$ & 123 & 41 & A7 \\
\hline Glyfada & Greece & Schmidt-Cassegrain & 0.28 & $V$ & 44 & 44 & A12 \\
\hline Guadarrama & Spain & Schmidt-Cassegrain & 0.25 & $B V R c$ & 27 & 41 & A15 \\
\hline Gualba & Spain & Schmidt-Cassegrain & 0.356 & $V(R I)_{C}$ & 3 & 1 & TA26 \\
\hline Horten & Norway & Ritchey-Chretien & 0.46 & $B V R_{b}$ & 11 & 4 & $\mathrm{~A} 21$ \\
\hline Jan Kochanowski & Poland & Schmidt-Cassegrain & 0.35 & $U B V R$ & 20 & 5 & A19 \\
\hline Las Pegueras & Spain & Schmidt-Cassegrain & 0.35 & V & 51 & 51 & A10 \\
\hline Leicester & United Kingdom & Corrected Dall-Kirkham & 0.508 & $B V R_{C}$ & 3 & 1 & A 25 \\
\hline Madrid-Ventila & Spain & Refractor & 0.06 & $V$ & 1 & 1 & A27 \\
\hline Magnago & Italy & Schmidt-Cassegrain & 0.25 & $B V I c$ & 15 & 5 & A20 \\
\hline Nerpio & Spain & Corrected Dall-Kirkham & 0.431 & $B V R_{C}$ & 34 & 32 & A14 \\
\hline New Mexico Skies & USA & Corrected Dall-Kirkham & 0.508 & $B V$ & 9 & 5 & A22 \\
\hline Rozhen & Bulgaria & Schmidt & 0.7 & $U B V(R I)_{C}$ & 50 & 10 & A 11 \\
\hline Rozhen & Bulgaria & Cassegrain & 0.6 & $B V(R I)_{C}$ & 23 & 7 & A 17 \\
\hline Slovak Academy & Slovakia & Cassegrain & 0.6 & $U B V(R I)_{C}$ & 139 & 30 & A6 \\
\hline Sonoita & USA & Folded Newtonian & 0.5 & $B V(R I)_{C}$ & 349 & 90 & $\mathrm{~A} 2$ \\
\hline Suhora & Poland & Cassegrain & 0.6 & $U B V R I$ & 307 & 57 & A3 \\
\hline Szczecin & Poland & Newton & 0.203 & $B V I$ & 66 & 34 & A8, A12 \\
\hline Tenerife-BRT & Spain & Schmidt-Cassegrain & 0.356 & $B V R I_{C}$ & 23 & 10 & A18 \\
\hline West Challow & United Kingdom & Schmidt-Cassegrain & 0.356 & $V I_{C}$ & 56 & 28 & A9 \\
\hline Wyspa Pucka & Poland & Schmidt-Cassegrain & 0.203 & $B V R_{C}$ & 172 & 72 & A4 \\
\hline IGeoE, Lisboa & Portugal & Schmidt-Cassegrain & 0.36 & $3840-7360 \AA^{R \sim 483}$ & 4 & 4 & \\
\hline Madrid-Ventila & Spain & Ritchey-Chretien & 0.2 & $3890-7600 \AA^{R \sim 600}$ & 1 & 1 & \\
\hline Rozhen & Bulgaria & Ritchey-Chretien $^{(*)}$ & 2.0 & $\mathrm{H}_{\alpha} *, \mathrm{H}_{\beta} *, \mathrm{Na}^{R \sim 16000}$ & 10 & 6 & \\
\hline Rozhen & Bulgaria & Ritchey-Chretien $^{(* *)}$ & 2.0 & $4000-9000 \AA^{R \sim 30000}$ & 2 & 2 & \\
\hline San Pedro Mártir & Mexico & Ritchey-Chretien & 2.1 & $5000-8600 \AA^{R \sim 2000}$ & 3 & 2 & \\
\hline West Challow & United Kingdom & Schmidt-Cassegrain & 0.28 & $3800-7585 \AA^{R \sim 1000}$ & 1 & 1 & \\
\hline
\end{tabular}

Notes. $N_{\mathrm{n}}$ is the number of observed nights; $N_{\mathrm{i}}$ is the number of individual brightness determinations summed over all the photometric bands. The last column presents the number of the table with the original data. $(*)$ are spectra made with Coude spectrograph and $(* *)$ are spectra made with ESperRo spectrograph.

\section{Observations}

Our call for observations (Gałan et al. 2014) gained a wide response from observers, using 30 telescopes located in Europe and two in North America. Several science facilities and organisations were involved in this campaign, as well as a large number of amateur astronomers, who were participating in an independent campaign coordinated by AAVSO (Waagen 2014). Technical details of the telescopes are listed in Table 1. All the telescopes were reflectors with a range from 0.2 to $2.0 \mathrm{~m}$ in diameter. More than 11000 individual photometric measurements were obtained with 27 instruments, most using $U B V(R I)_{C}$ passbands of Johnson-Cousins photometric system and in several cases $R I$ filters closer to Johnson's realisation of these bands. The photometric data cover 243 days from March 22 to November 20, 2014.

One photometric measurement in near-infrared $J H K_{S}$ bands was obtained on June 3, 2010 (HJD=2455350.67) with the CAIN infrared camera operating on $1.5 \mathrm{~m}$ Carlos Sánchez telescope at Teide Observatory. The frames were reduced on flatfields, bad pixel corrected, background subtracted, and finally combined with the use of CAINDR ${ }^{1}$ data reduction tasks

1 CAINDR - Infrared Camera Reduction Software developed by R. Berrena and J. Acosta - IAC v0.5 - July 2007. working in the IRAF $^{2}$ environment; the photometry was performed using the apphot package. The resulted magnitudes are shown in Table A28.

\subsection{Transformation to the standard system}

We proposed to use the four brightest stars from the Meinuger's sequence (Meinunger 1975) as comparison and check stars; all these objects are close in the sky, within $\sim 3^{\prime}$ to the position of EE Cep (Gałan et al. 2014). These stars were denoted as " $a$ ", " $b$ ", " $c$ ", and " $d$ " for BD+55 2690, GSC-39732150, BD+55 2691, and GSC-39731261, respectively (Fig. 2).

Most of the data were expressed by the observers directly as apparent magnitudes. In these cases we calculated differential magnitude with respect to " $a$ " star: $(v-a)$. In other cases, differential magnitudes were already given with respect to " $a$ " star. All data were corrected for the differences resulting from using different instruments. We chose two standard systems.

2 IRAF is distributed by the National Optical Astronomy Observatories, which are operated by the Association of Universities for Research in Astronomy, Inc., under a cooperative agreement with the National Science Foundation. 


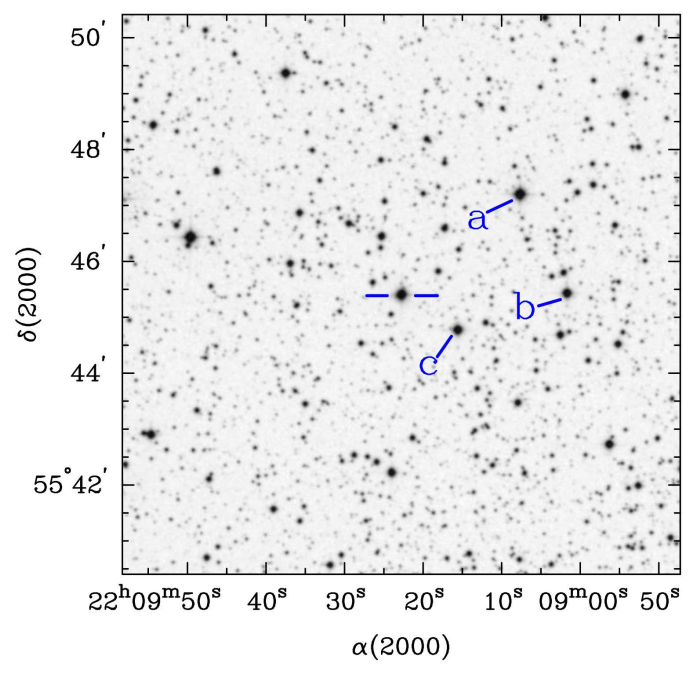

Fig. 2. $10^{\prime} \times 10^{\prime}$ DSS-2-red finding chart for EE Cep.

The photometric data from Suhora Observatory (Table A3) were adopted as a zero point for URI bands in the Johnson system. These data covered, with a good density of observational points in time, the whole time duration of the eclipse and a long baseline before and after the eclipse. For $B V(R I)_{C}$ bands in JohnsonCousins system, we chose photometric data from the University of Athens Observatory (UOAO, see Table A1). The data consists of 10-50 individual observations every night during the entire duration of the eclipse (descending and ascending wings, as well as outside the eclipse). There are differences between the Johnson $R I$ bands and Johnson-Cousins $(R I)_{C}$ bands, thus we used different magnitudes of " $a$ " star (Table 2). For the Johnson system, we used magnitudes of " $a$ " star given by Mikołajewski et al. (2003), and for Johnson-Cousins system we used magnitudes measured by the UOAO (Table 2). We decided to reject differential measurements which had been done with respect to " $b$ " and " c" stars. According to Samus et al. (2009) these are suspected to be variable stars. We mentioned in Gałan et al. (2014) that those stars were monitored in a time period of about five years and also during and close to EE Cep eclipse in 2008-2009. The variability of " $b$ " star was not confirmed. However, we did not want any unexpected variability from this star in the course of the eclipse in 2014.

As in the case of previous two campaigns, we transformed measurements to one standard system in every band. The procedure was similar to Gałan et al. (2012), but this time the eclipse was deeper and we had to take the effect of the colours into consideration. The difference between the two systems is not constant as a function of depth. We assumed that the relation between the shift among standard system and the instrumental system $\left(v_{r}-v\right)$ and depth of eclipse can be approximated by a linear function. This relation takes the form of $f(x)=A x+B$, where $x$ is the depth of eclipse measured from the point where $(v-a)=0$ and $f=\left(v_{r}-v\right)$ is the shift between standard system $v_{r}$ and shifted system $v$. This was calculated by taking measurements that had been done at the same time in different systems. We assumed that observations which were done at time duration shorter than 0.25 day fulfilled this condition. Parameters $A$ and $B$ were estimated by fitting the linear function to the data. We obtained instrumental data shifted to zero point by subtracting calculated shift $v-f(v)$. We started from the standard system data set, which had the largest number of averaged measurements. As a next step, we added less numerous data sets, ending
Table 2. Magnitudes of star $\mathrm{BD}+55^{\circ} 2690$ in $U B V R I(R I)_{C}$ bands.

\begin{tabular}{ccccccc}
\hline \hline$U^{(*)}$ & $B^{(* *)}$ & $V^{(* *)}$ & $R_{C}{ }^{(* *)}$ & $I_{C}{ }^{(* *)}$ & $R^{(*)}$ & $I^{(*)}$ \\
\hline 10.86 & 10.704 & 10.397 & 10.218 & 10.015 & 10.09 & 9.87 \\
\hline
\end{tabular}

Notes. ${ }^{(*)}$ Given by Mikołajewski et al. (2003). ${ }^{(* *)}$ Given by the UOAO.

up to the data sets with two measurements. At the end, all photometric data from Tables A1 to A27 were averaged to one point per night. Table A29 contains this average values with standard deviation and number of measurements used to average.

\subsection{Spectroscopic data}

High-resolution spectra were collected with two spectrographs (Coudé and ESpeRo) operating on the $2 \mathrm{~m}$ Ritchey-Chretién telescope at the Rozhen Observatory, Bulgaria. Two spectra $(R \sim 30000)$ in range $\sim 4000-9000 \AA$ were obtained with ESpeRo echelle spectrograph (Bonev et al. 2017). Ten spectra $(R \sim 16000)$ covering narrow ranges $(\sim 200 \AA)$ were obtained with Coudé spectrograph during six nights in the period from April 4 to August 6, 2014. Balmer $\mathrm{H}_{\alpha}, \mathrm{H}_{\beta}$, and $\mathrm{H}_{\gamma}$ and sodium $\mathrm{Na}$ I doublet line profiles from these spectra are shown in Fig. 3. Nine low-resolution spectra were collected with four instruments in the range $\sim 4000-8600 \AA$. All spectra were heliocentric corrected and normalised to the continuum. The list of spectra and instruments together with some additional information are given in Table 1 and all obtained spectra are available as FITS files at the CDS.

\section{Results}

\subsection{Photometric results}

The best coverage with observations was obtained in the $V$ band used by every observer. Coverage in $R_{C}$ and $I_{C}$ is also very dense. After averaging observations in time intervals as described in Sect. 2.1, we obtained useful data on 170 individual nights. The final $U B V R I(R I)_{C}$ light curves are presented in Fig. 4.

The minimum of the eclipse took place on JD $=2456894.0 \pm$ 0.05 . The timing residual (observations minus calculations; i.e. the $(\mathrm{O}-\mathrm{C})$ value) with respect to ephemeris from Eq. (1) is small $(\mathrm{O}-\mathrm{C}=0.75)$. The amplitude of the eclipse reached from about 0.81 to 0.61 in $U$ and $I_{C}$ bands, respectively. The amplitudes in all bands and the averaged differential magnitudes $(\overline{v-a})$ outside the eclipse (to phase $\sim-0.054$ and from phase $\sim 0.024$ ) are shown in Table 3.

The profiles of the eclipse in all bands are similar to those from 2003 and 2008-2009 ( $E=9$ and $E=10)$. In particular, there was a "bump" at about nine days before the photometric minimum during two previous eclipses. During this eclipse event $(E=11)$ it is not as evident in the light curve as in the all other previous deep eclipses, but it is visible in the colour indices ( $B-I_{C}, V-I_{C}$, and $B-R_{C}$ ) that we calculated (Fig. $4-$ bottom). Neglecting the second order effects, i.e. short and small amplitude variations, as observed during both previous campaigns and interpreted as a manifestation of the possible complex, multiring structure of the disc (Gałan et al. 2010), the observed colour indices generally show a two-component profile with a maximum of the "bump" on roughly nine to ten days before the photometric minimum. The beginning of the fast ingress can be observed in all observed photometric bands. A small ( $\left.\sim 0^{\mathrm{m}} 05\right)$ 

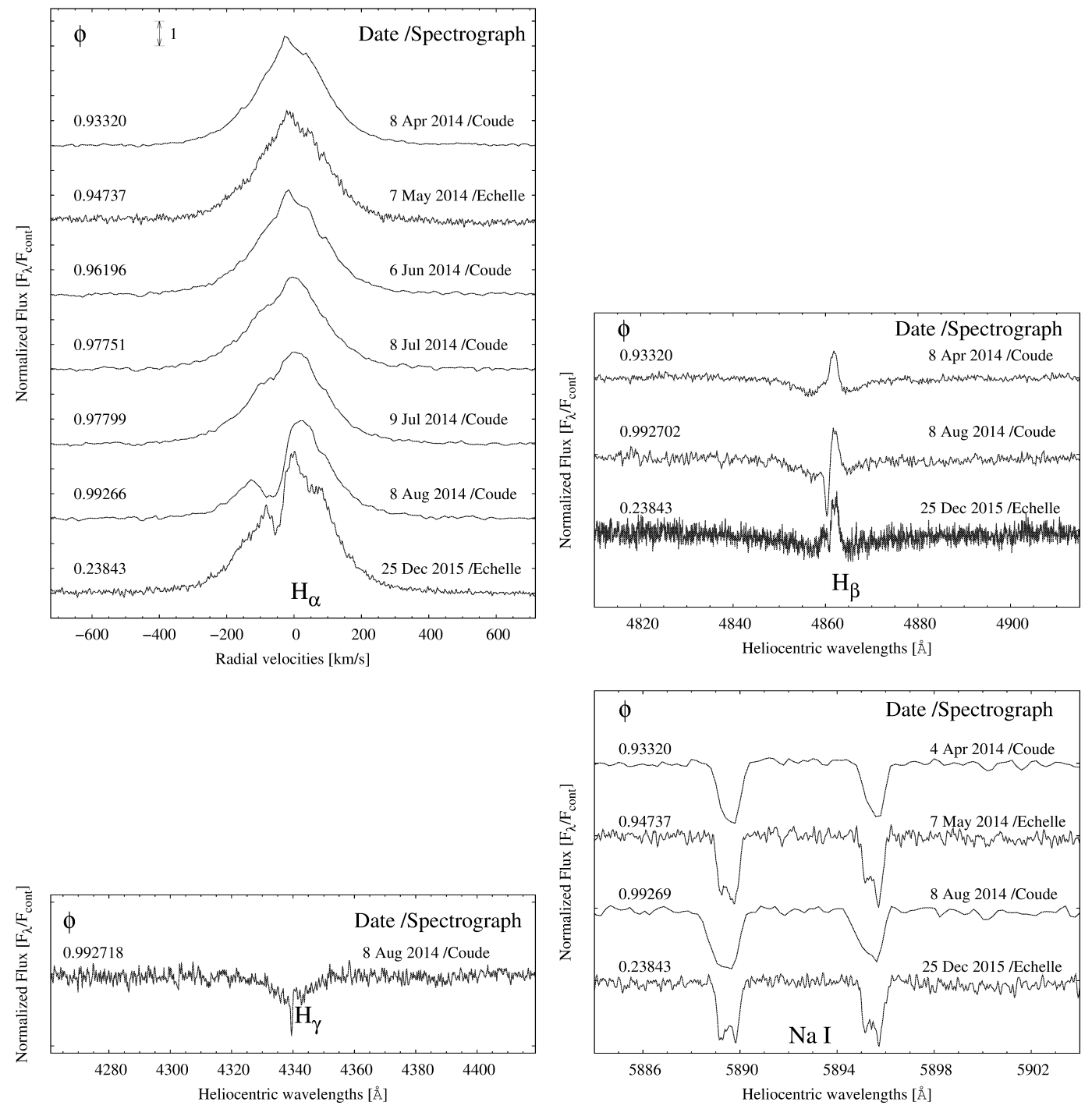

Fig. 3. Balmer $\mathrm{H}_{\alpha}, \mathrm{H}_{\beta}$, and, $\mathrm{H}_{\gamma}$, and $\mathrm{Na}$ I doublet line profiles in the high-resolution spectra obtained during and around the eclipse EE Cep in 2014 $(E=11)$.

dip is also present, which is visible in $V$ and $B$ light curves at orbital phase $\sim-0.015$ (Figs. 7 and 4 ). This coincides roughly in phase and amplitude, which are subject to change slightly because of changes in the orientation of the disc; this dip was noticed during the eclipses of previous campaigns in the $B$ and $V$ light curves, but corresponding events observed previously roughly at a similar time after the mid-eclipse were not clearly detectable. The number of measurements collected in the very near-infrared domain $I$ and $I_{C}$ bands during this campaign turned out, unfortunately, to be too small to confirm the repeatable brightening by several 100ths of magnitudes with a maximum at orbital phase $\sim 0.2$, which we reported after previous campaigns (Gałan et al. 2012). However, the independent campaign by AAVSO collected a relatively large number of measurements in the $I$ band. There are significant differences between photometric systems, and the scatter is large, but after correcting for them in the $I$-band light curve for epoch $E=11$ there also seems to be an apparent maximum at phase $\sim 0.2$ (see Fig. 5).

Our $J H K_{S}$ photometry obtained at phase $(\phi=0.247)$, during the time of fall after reaching the maximum brightness, compared to 2MASS photometry obtained at $\phi=0.338$ (Table A28) shows a slightly increased brightness coherent with the $I$ band.
This confirms the reality of this phenomenon and indicates that we can expect a somewhat increased amplitude of this effect in the near-infrared region, with a maximum falling somewhere in the region of the $H$ band.

\subsection{Calculating the new ephemeris}

The eclipses of EE Cep are asymmetric, so we could not use standard methods to calculate the time of minimum in the light curve. We decided to use the algorithm described by Kwee \& van Woerden (1956). The same method was used by Mikołajewski \& Graczyk (1999) to calculate the minimum of epoch $E=4$ eclipse, and this helped to calculate the minima of epochs 2, 5, and 8 and also to calculate the minima of the last three eclipses (epochs 9, 10, and 11). Using every known time of minimum for EE Cep (Table 4), we calculated the O-C residuals for the times of minima using the linear ephemeris $\mathrm{JD}($ Min $)=2434344.1+2049 \mathrm{~d} .94 \times E$ by Mikołajewski $\&$ Graczyk (1999). These values are listed in Table 4 and denoted in Fig. 6. The best linear fit to the residuals gives the new ephemeris

$\mathrm{JD}(\operatorname{Min})=2434345.16( \pm 2.08)+2049.78( \pm 0.22) \times E$. 


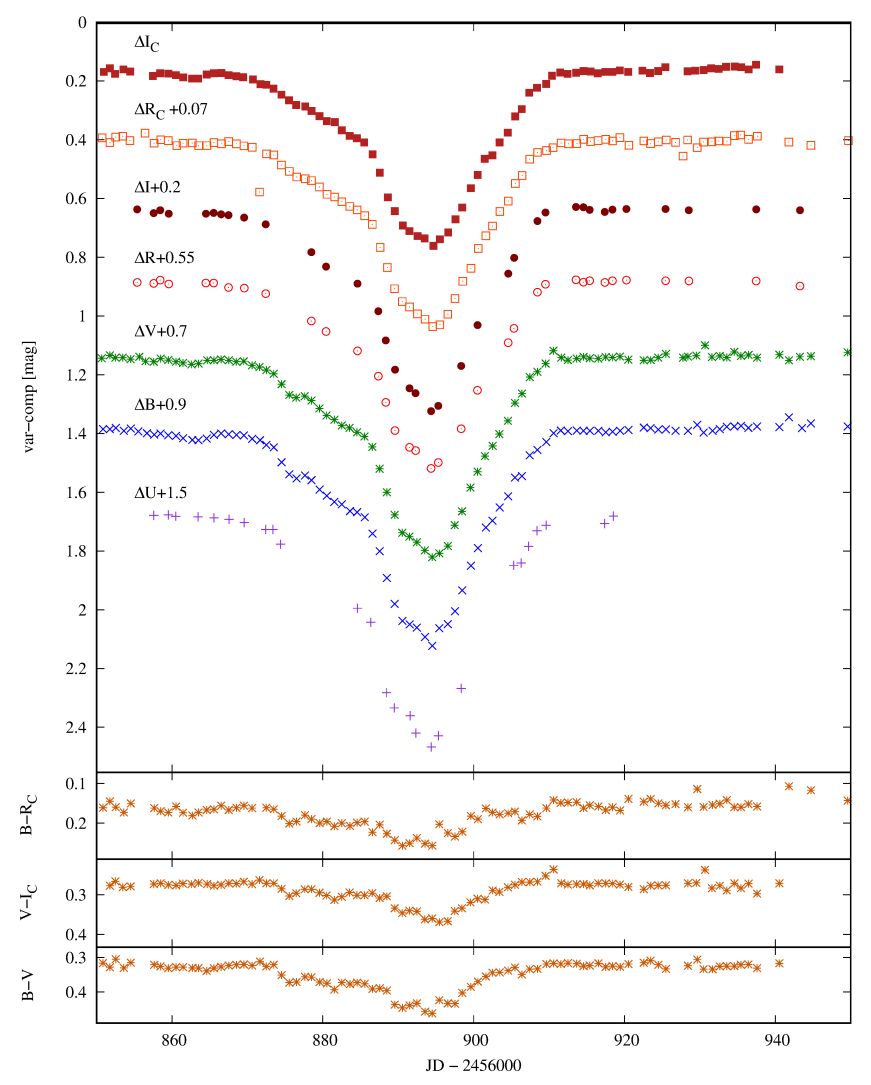

Fig. 4. Average points from Table A29 obtained from photometric measurements of the 2014 eclipse. The $U B V R I(R I)_{C}$ light curves (top) and three colour indices (bottom) are presented.

Table 3. Average magnitudes of the EE Cep outside the eclipse (to phase $\sim-0.054$ and from phase $\sim 0.024$ ), brightnesses in the minimum of the eclipse, and the eclipse depths (differences between the outside and the minimum values) for seven particular passbands.

\begin{tabular}{lccccccc}
\hline \hline Band & $U$ & $B$ & $V$ & $R_{C}$ & $I_{C}$ & $R$ & $I$ \\
\hline Outside & 0.12 & 0.48 & 0.43 & 0.33 & 0.15 & 0.31 & 0.41 \\
Minimum & 0.97 & 1.22 & 1.12 & 0.97 & 0.76 & 0.97 & 1.12 \\
Depth & 0.83 & 0.75 & 0.71 & 0.65 & 0.62 & 0.66 & 0.71 \\
\hline
\end{tabular}

\subsection{Spectroscopic results}

We did not observe any significant changes in the $\mathrm{H}_{\alpha}$ line up to July 8 and July 9, 2014, when signs of absorption components began to appear on the blue wings of the emission component (Figs. 3 and 7). This coincides with the time when the small dip starts to develop in the light curves, which signifies the beginning of the photometric eclipse. The new precise photometric data with dense time coverage reveals more subtle changes and a more accurate determination of the eclipse times and their total duration. The shallow eclipses last up to 2.5-3.5 months and this means there is less of a difference from results obtained from spectroscopic observations. The total duration times estimated from the old photometric data (Graczyk et al. 2003) seem to be underestimated. The Balmer $\mathrm{H}_{\alpha}, \mathrm{H}_{\beta}$ and $\mathrm{H}_{\gamma}$ profiles in the spectra obtained deep in the eclipse (Aug 8, 2014) show deep absorption component on their blue wings. Interestingly, $\mathrm{H}_{\alpha}$ and $\mathrm{H}_{\beta}$ lines in the spectra obtained on December 25, 2015 also show an absorption component; this is the orbital phase $\sim 0.24$ during

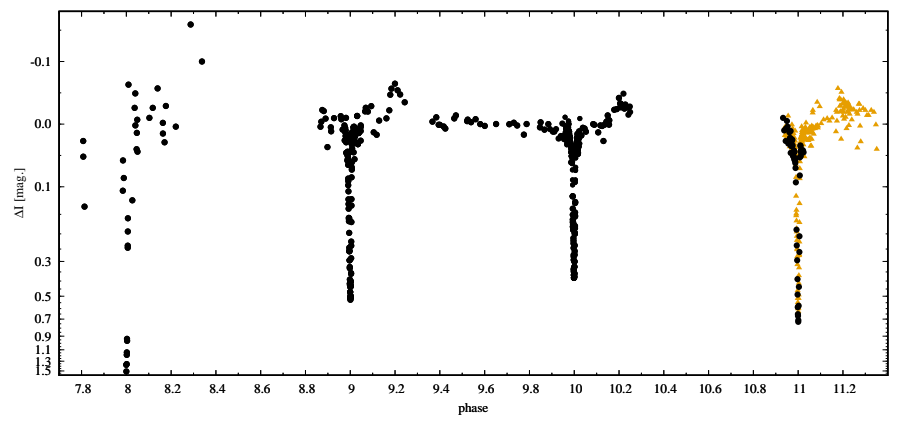

Fig. 5. Differential $I$ magnitudes of EE Cep during and around of eclipses at epochs $E=8,9,10$, and 11 . The zero value represents average brightness outside eclipses (phase 0.4-0.8). The yellow triangles show the AAVSO I-band light curve after correcting for the differences between photometric systems of particular observers. The magnitudes of epoch $E=11$ (black points) are the same as in Fig. 4 for the $I$ band.

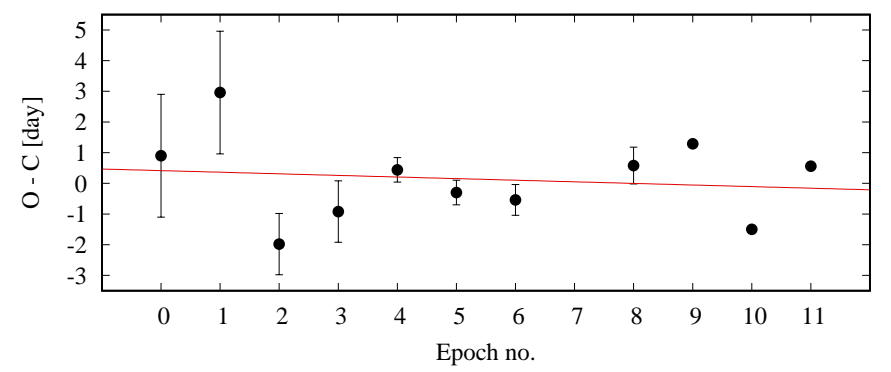

Fig. 6. O-C diagram with a linear fit (solid line) to residuals.

Table 4. Observed moments of minima and the O-C (observations minus calculations) residuals calculated according to the linear ephemeris $\mathrm{JD}(\mathrm{Min})=2434344.1+2049.94 \times E$ (Mikołajewski \& Graczyk 1999).

\begin{tabular}{lcccl}
\hline \hline Epoch & Light & JD & O-C & Reference \\
\hline 0 & $\mathrm{mpg}$ & $2434345.0 \pm 2.0$ & 0.90 & {$[1]$} \\
1 & $\mathrm{mpg}$ & $2436397.0 \pm 2.0$ & 2.96 & {$[1]$} \\
2 & $\mathrm{mpg}$ & $2438442.0 \pm 1.0$ & -1.98 & {$[2]$} \\
3 & $\mathrm{mpg}$ & $2440493.0 \pm 1.0$ & -0.92 & {$[3]$} \\
4 & $\mathrm{~V}$ & $2442544.3 \pm 0.4$ & 0.44 & {$[2]$} \\
5 & $\mathrm{~V}$ & $2444593.5 \pm 0.4$ & -0.30 & {$[2]$} \\
6 & $\mathrm{~V}$ & $2446643.2 \pm 0.5$ & -0.54 & {$[4]$} \\
7 & $\mathrm{~V}$ & $2448691.87 \pm 0.89$ & -1.78 & {$[5]$} \\
8 & $\mathrm{~V}$ & $2450744.2 \pm 0.6$ & 0.58 & {$[2]$} \\
9 & $\mathrm{~V}$ & $2452794.85 \pm 0.14$ & 1.29 & This work \\
10 & $\mathrm{~V}$ & $2454842.0 \pm 0.1$ & -1.50 & This work \\
11 & $\mathrm{~V}$ & $2456894.0 \pm 0.2$ & 0.56 & This work \\
\hline
\end{tabular}

References. ${ }^{[1]}$ Meinunger (1973); ${ }^{[2]}$ Mikołajewski \& Graczyk (1999); ${ }^{[3]}$ Baldinelli et al. (1975); ${ }^{[4]}$ Di Luca (1988); ${ }^{[5]}$ Halbach (1992).

the brightening event (soon after its maximum) in the $I$ band. The blue-shifted absorption components are visible also in $\mathrm{Na}$ I doublet observed during this phase. Sodium develops absorption before and after the external photometric contacts of the eclipses (e.g. spectrum on May 7, 2014). Similar behaviour of the sodium and potassium lines was observed in $\varepsilon$ Aur system (see e.g. Leadbeater et al. 2012; Tomov et al. 2012). Several lowresolution $(R \sim 500-1000)$ spectra listed in Table 1 generally confirm the evolution of the EE Cep spectrum shown by Boyd (2015). 


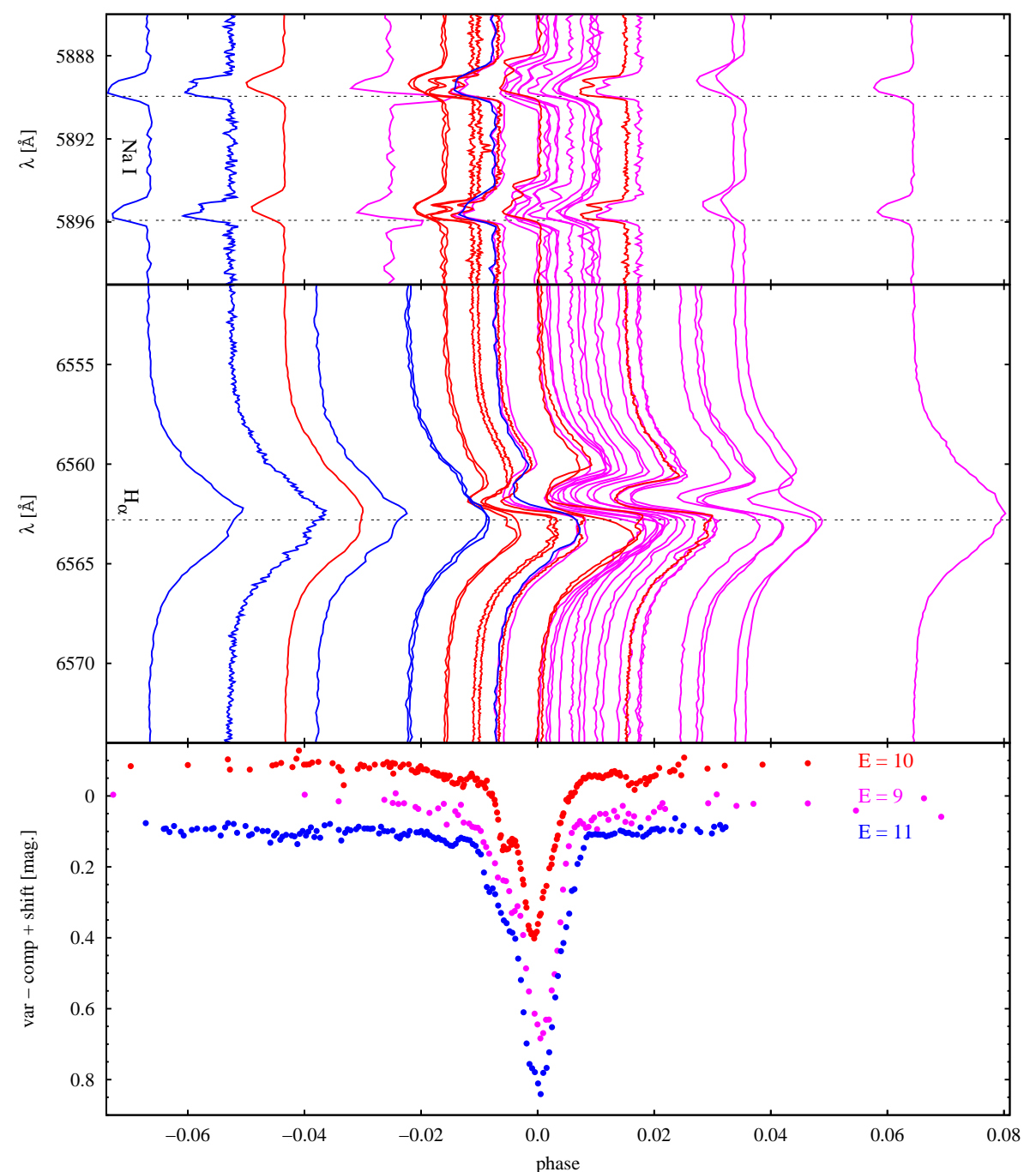

Fig. 7. Evolution of the Na I line profiles (top), $\mathrm{H}_{\alpha}$ line profiles (middle), and $B$ light curves (bottom) during and around of eclipses at epochs $E=9,10$, and 11 . Data are folded according to ephemeris by Eq. (1).

\section{Precessing Be star model}

Analysis of the photometric data of the last campaigns together with the changes in the spectral line profiles proves that the eclipses have longer total duration, even up to $\sim 3.5$ months, than postulated on the basis of observations of previous eclipses. The last eclipse reached a depth of only $\sim 0 \mathrm{~m} .75 \mathrm{mag}$ in $B$, which is much shallower than expected $\sim 2 \mathrm{~m} 0$ according to the Gałan et al. (2012) model. One possible reason could be that the assumptions made to the disc precession model were too simple: in particular, (i) the circularity of the orbit and (ii) a very simplified, disc density profile $\left(\sim r^{-2}\right)$. Moreover, (iii) the disc diameter was adopted quite arbitrarily as not larger than $D \sim 150 R_{\odot}$. This could be justified by making simple assumptions about the system geometry shown on Fig. 8. The disc size can be calculated as a function of component separation $a$, companion radius $R_{S}$, and viewing angle $\theta$. The viewing angle corresponds to half the duration of the eclipse, which is about three months, thus $\theta \simeq 0.056$. We assumed that disc mass is much lower than the Be star mass (which is $\sim 8 M_{\odot}$ according to Gałan et al. 2012 and $R_{S} \simeq 9 R_{\odot}$ ). Taking this into consideration the component separation determined by Keplerian law is $a=1360 R_{\odot}$, which gives the disc diameter about $370 R_{\odot}$. However this estimation does not take into the impact parameter $D$, inclination and eccentricity of the system into consideration.

In future work, we will take another model that could explain changes in eclipse depths into consideration. At the moment, the

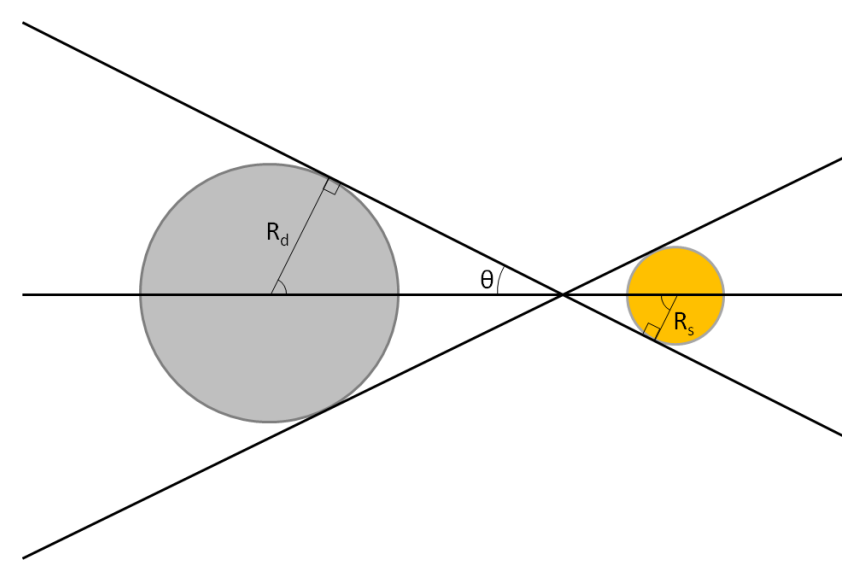

Fig. 8. Simple assumptions about geometry of the EE Cep system.

hypothesis of eclipses caused by a disc still offers the best way to explain the grey character of all eclipses and that the secondary component is still eluding detection. This is also a good explanation for small changes in the light curve in the early stage of the eclipses.

We want to propose a model in which the Be star is precessing instead of the disc. In this case, the changes in the eclipse depth would be caused by eclipsing the hot spots on the poles of the star as shown in the diagram in Fig. 9. We can distinguish 

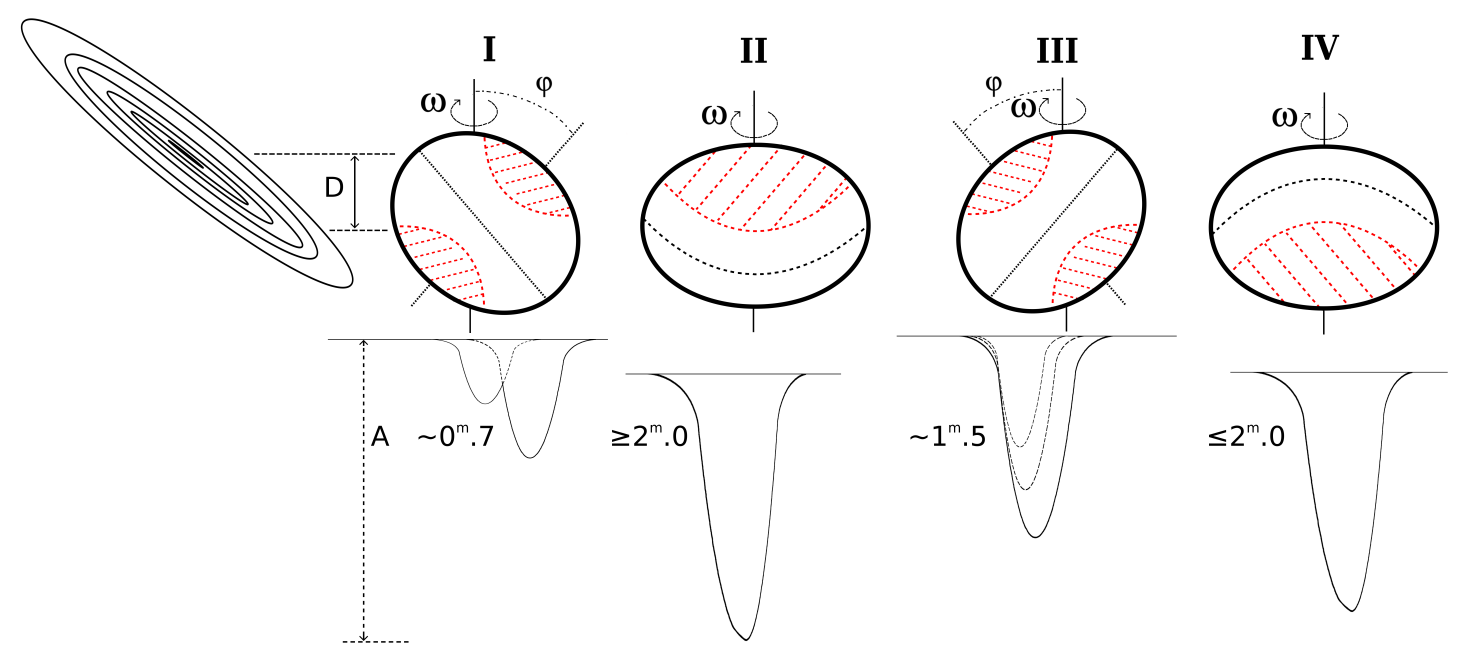

Fig. 9. Precessing Be star model explaining changes in the depths of the eclipses.

four special orientations in the geometry of the system, in which the angle of the disc and impact parameter $D$ is the same. Only the inclination of the star $\varphi$ changes owing to precession, which would cause different amplitude $A$ of the eclipses. The four orientations are as follows:

I. Two clearly separate, relatively shallow minima with depth $\sim 0$. 7 (epochs $9,10,11$ ). The occultation of the bottom pole is first followed by the top pole.

II. Only the visible hot pole is being highly eclipsed (epochs $0,1,2)$. The hot spot is close to the disc centre. Deep $(A \gtrsim 2$. 0$)$ eclipses are observed.

III. Overlapping minima from two poles that are being eclipsed almost simultaneously (epochs $4,5,6)$. The hot spot caused by the bottom pole experiences a weaker eclipse $(A \sim$ $1 \mathrm{~m}$ 5) through the outer, more transparent parts of the disc. Intermediate depth eclipses are observed.

IV. The hot spot, further from the disc centre is eclipsed (epoch 8); this is similar to case II but the eclipse is less deep $A \lesssim 2$. 0 .

Changes in the inclination of Be star (precession) may cause small changes in its brightness and colours due to the visibility of hot polar regions. Line broadening may also change during the precession cycle. Our future studies will concentrate on verifying this model.

\section{Concluding remarks}

In this work, we present the processed photometric and spectroscopic data that have been obtained during the recent eclipse season of EE Cep in 2014 ( $E=11)$. Several conclusions might explain the most important observational features of the eclipses which should be taken into account.

There are small effects detectable in the light and colour curves which could be related to a possible complex multi-ring structure of the disc. Gałan et al. (2010) speculate that possible planets could be responsible for the formation of the gaps in the disc. However, this adds an extra complication to the model and such small second-order effects should be neglected in the modelling of the precession.

There are indications that the orbit is significantly eccentric. We believe that variations out of the eclipses noted in I-band light curves at orbital phase $\sim 0.2$ (Gałan et al. 2012, and this paper) could be related to proximity effects when components are approaching each other close to periastron passage. In that case, the acceleration in the orbital motion may be an additional reason for the observed eclipse asymmetry and this complication may have to be taken into account in the model.

The most serious problem for developing the unified model of all eclipses are two events at epochs $E=3$ and $E=8$, which are characterised with extremely different depths from neighbouring eclipses. In the case of the disc precession model, this effect could be reproduced by realisation of the scenario in which two effects compete when the disc is seen nearly edge-on: (i) the rapid change in the optical depth of the disc; and (ii) the change in size of the disc projection, which makes changes in the extent of obscuration (see Gałan et al. 2012, Sect. 4.4). The larger disc diameter should enhance the efficiency of this mechanism because this would imply a smaller inclination of the precession axis. In the alternative option of the star precession some other mechanism explaining such quick changes will have to be proposed; for example, it could be considered to what extent the slope of the precession axis of a star could be responsible for this.

Applying new modelling techniques might bring a breakthrough. For example, any possibility to know parameters of the orbit from measurements of the radial velocity variations (very difficult for such a rapidly rotating, hot star) and/or if possible the movement of the cold component from infrared interferometry, may be crucial for constraining the parameters and understanding of this system. Our still scant infrared data ( $I J H K$ bands) seem to indicate the possibility to detect the cold component through systematic infrared monitoring during the whole orbital cycle.

Acknowledgements. This study has been partly supported by the Polish National Science Centre grant No DEC-2015/19/D/ST9/02974. This paper is partly a result of the exchange and joint research project Spectral and photometric studies of variable stars between Polish and Bulgarian Academies of Sciences. A large number of photometric data on EE Cep were collected during the photometric monitoring observations with the robotic and remotely controlled observatory at the University of Athens Observatory - UOAO (Gazeas 2016). This work was partially supported by VEGA 2/0031/18 and APVV-15-0458 projects. We would like to thank all the technical staff that made the observations possible. ZK, DMo and AP were supported by the NCN grant No. 2016/21/B/ST9/01126. 


\section{References}

Baldinelli, L., Ghedini, S., \& Tubertini, C. 1975, IBVS, 1009 Bonev, T., Markov, H., Tomov, T., et al. 2017, Bulg. Astron. J., 26, 67 Boyd, D. 2015, J. Br. Astron. Assoc., 125, 94

Di Luca, R. 1988, Giornale dell' Assoc. Astrofili Bologna, 92, 5

Gaia Collaboration (Prusti, T., et al.) 2016, A\&A, 595, A1

Gaia Collaboration (Brown, A. G. A., et al.) 2018, A\&A, 616, A1

Gałan, C., Mikołajewski, M., Tomov, T., et al. 2008, IBVS, 5866

Gałan, C., Mikołajewski, M., Tomov, T., et al. 2010, ASP Conf. Ser., 435, 423

Gałan, C., Mikołajewski, M., Tomov, T., et al. 2012, A\&A, 544, A53

Gałan, C., Wychudzki, P., Mikolajewski, M., et al. 2014, IBVS, 611

Garrido, H. E., Mennickent, R. E., Djurašević, G., et al. 2016, MNRAS, 457, 1675

Gazeas, K. 2016, Rev. Mex. Astron. Astrofís., 48, 22

Guinan, E. F., \& Dewarf, L. E. 2002, ASP Conf. Ser., 279, 121

Graczyk, D., Mikołajewski, M., Tomov, T., et al. 2003, A\&A, 403, 1089

Hajduk, M., Zijlstra, A. A., \& Gęsicki, K. 2008, A\&A, 490, 7

Halbach, E. A. 1992, J. Am. Assoc. Var. Star Obs., 21, 129

Kenworthy, M. A., \& Mamajek, E. E. 2015, ApJ, 800, 126

Kwee, K. K., \& van Woerden, H. 1956, Bull. Astron. Inst. Neth., 12, 327

Leadbeater, R., Buil, C., Garrel, T., et al. 2012, J. Am. Assoc. Var. Star Obs., 40, 729

Lipunov, V., Gorbovskoy, E., Afanasiev, V., et al. 2016, A\&A, 588, A90

Meinunger, L. 1973, Mitt. Verän. Sterne, 6, 89

Meinunger, L. 1975, IBVS, 965

Mikołajewski, M., \& Graczyk, D. 1999, MNRAS, 303, 521

Mikołajewski, M., Tomov, T., Graczyk, D., et al. 2003, IBVS, 5412

Miszalski, B., Mikołajewska, J., Köppen, J., et al. 2011, A\&A, 528, A39

Rattenbury, N. J., Wyrzykowski, Ł., Kostrzewa-Rutkowska, Z., et al. 2015, MNRAS, 447, 31

Romano, G. 1956, Coelum, 24, 135

Samus, N. N., Kazarovets, E. V., Durlevich, O. V., Kireeva, N. N., \& Pastukhova,

E. N. 2009, General Catalogue of Variable Stars (Samus+, 2007-2017)

Scott, E. L., Mamajek, E. E., Pecaut, M. J., et al. 2014, ApJ, 797, 6

Tomov, T., Wychudzki, P., Mikołajewski, M., et al. 2012, Bulg. Astron. J., 18,

von Zeipel, H. 1924, MNRAS, 84, 665

Waagen, E. O. 2014, AAVSO Alert Notice, 502

Weber, R. 1956, Doc. des Obs. Circ., 9

1 Nicolaus Copernicus Astronomical Centre PAS, Warsaw, Poland e-mail: dapien@camk.edu.pl, cgalan@camk.edu.pl

2 Nicolaus Copernicus University, Toruń, Poland

3 Section of Astrophysics, Astronomy and Mechanics, Department of Physics, National and Kapodistrian University of Athens, 15784 Zografos, Athens, Greece

${ }^{4}$ Sonoita Research Observatory/AAVSO, USA

5 Astronomical Observatory, Jagiellonian University, Cracow, Poland

6 Mount Suhora Astronomical Observatory, Cracov Pedagogical University, ul. Podchorazych 2, 30-084 Cracow, Poland
${ }^{7}$ Institute of Astronomy and National Astronomical Observatory, Bulgarian Academy of Sciences, Sofia, Bulgaria

8 Department of Physics, Shumen University, Shumen, Bulgaria

9 PTMA, Szczecin, Poland

10 Furzehill House, Ilston, Swansea SA2 7LE, UK

11 Instytut Astronomiczny, Uniwersytet Wrocławski, Poland

12 Club d'astronomie de Mont-Bernenchon, France

13 BAA Variable Star Section, West Challow Observatory, Oxfordshire, UK

14 Department of Physics and Astronomy, York University, Toronto, Ontario, Canada

15 Instituto de Astronomía, Universidad Nacional Autonoma de Mexico, Ensenada, BC, Mexico

16 Observatorio Las Pegueras, NAVAS DE ORO (Segovia), Spain

17 Hellenic Amateur Astronomy Association, Athens, Greece

18 Astronomical Institute, Slovak Academy of Sciences, Tatranská Lomnica, Slovak Republic

19 ELTE Eötvös Loránd University, Gothard Astrophysical Observatory, 9700 Szombathely, Hungary

20 MTA-ELTE Exoplanet Research Group, Szent Imre h. u. 112, 9700 Szombathely, Hungary

21 Guadarrama Observatory MPC458, Madrid, Spain

22 Center for Astrophysics, Harvard \& Smithsonian, 60 Garden Street, Cambridge, MA, USA

23 Astrolab Iris, Zillebeke Belgium, Belgium

24 Institute of Physics, Astrophysics Division, Jan Kochanowski University, Kielce, Poland

25 Stazione Astronomica Betelgeuse, Magnago, Milano, Italy

26 Private Observatory, Maladziechna, Belarus

27 The Faculty of Mathematics and Natural Sciences, Department of Physics, University of Oslo, Oslo, Norway

${ }^{28}$ Cerro del Viento Observatory (MPC I84), Badajoz, Spain

29 Observatorio do Instituto Geografico do Exercito - Lisboa, Lisboa, Portugal

30 AstroCamp Observatory (MPC I89), Nerpio, Spain

31 Institute of Physics, Faculty of Science, Ss. Cyril and Methodius University, Skopje, FYR of Macedonia

32 AAVSO, Cambridge, MA 02138, USA

${ }^{33}$ Groupe Européen d'Observations Stellaires (GEOS), Bailleau l'Evêque, France

34 Private Observatory Madrid-Ventilla MPC J30, Spain

35 Gualba Observatory, Spain

36 Department of Physics and Astronomy, University of Leicester, Leicester, UK

37 Menke Scientific, Barnesville, MD 20838, USA

38 Amateur society "Astroblocknote", Minsk, Belarus

39 Mont Mégantic Observator, University of Montreal, Montreal, Canada 